\title{
Drug induced pulmonary parenchymal disease
}

\author{
Rajendra Prasad*, Pawan Gupta, Abhijeet Singh, Nitin Goel \\ Department of Respiratory Medicine, Vallabhbhai Patel Chest Institute, University of Delhi, Delhi, India.
}

\begin{abstract}
Summary Drug-induced pulmonary parenchymal disease (DIPPD) can be caused by a variety of agents, including antibiotics, chemotherapeutic drugs, antiarrhythmic agents and nonsteroidal anti-inflammatory drugs (NSAIDs). DIPPD includes acute bronchospasm, organizing pneumonia, alveolar hypoventilation and hypersensitivity reactions. History, physical examination and investigations are required mainly to exclude other causes of lung diseases. Investigations may include chest radiography, pulmonary function testing and bronchoscopy with bronchoalveolar lavage (BAL). Recognition of DIPPD is difficult because the clinical, radiologic, and histologic findings are nonspecific. Management includes drug withdrawal and in some cases corticosteroid therapy. In this article we reviewed the various drugs known to cause pulmonary parenchymal diseases, various patterns of parenchymal diseases observed and their management.
\end{abstract}

Keywords: Pulmonary disease, bronchospasm, organizing pneumonia

\section{Introduction}

Pulmonary toxicity has been linked to increasing number of drugs since the late 19th century. With the advancement in development of new drugs, more drugs are now associated with lung diseases. In 1880, Osler described heroin-induced pulmonary edema during an autopsy (1). Drugs can affect any part of lung but parenchyma is most frequently involved. Currently, more than 350 agents are associated with drug induced pulmonary parenchymal diseases (DIPPD) $(2,3)$. While uncommon, pulmonary adverse reactions often lead to significant physiologic impairment and/or necessitate intervention (4). Although drug-induced pulmonary diseases (DIPPD) vary in their pathophysiology, presentation and prognosis but diagnosis and treatment approach is similar in all groups. DIPPD is primarily a diagnosis of exclusion. Proper diagnosis requires a high index of suspicion and familiarity with the clinical syndromes associated with DIPPD. The present article aims to review the various drug induced pulmonary parenchymal diseases and their management.

\footnotetext{
*Address correspondence to:

Dr. Rajendra Prasad, Vallabhbhai Patel Chest Institute, University of Delhi, Delhi, India.

E-mail: rprasadkgmc@gmail.com
}

\section{Pathogenesis}

Drug induced pulmonary parenchymal disease generally involves pulmonary parenchyma but can also involve the airways in addition. Mechanisms include direct oxidative injury, cytotoxic effects on alveolar capillary endothelial cells, immune-mediated lung injury and amphophilic medications causing deposition of phospholipid within the cells. These result in a variety of clinical and histologic patterns including bronchospasm, pneumonitis, pulmonary fibrosis, hypersensitivity reaction and non-cardiogenic pulmonary edema that has been mentioned in Table 1 .

\subsection{Bronchospasm}

Drug induced bronchospasm can be triggered by any drug delivery route (aerosol, oral, IV or topical). Acute bronchospasm may be seen within few minutes of drug administration. Symptoms may manifest as wheezing and shortness of breath. Chest radiographs may show hyperinflation. Drugs cause bronchoconstriction by direct effect on airway smooth muscle by $\beta$ blockers (5) and anticholinergic (6), inhibitory action on cyclooxygenase by aspirin (7), irritation of airways via a vagal reflex by ipratropium bromide and cromoglycate (8), release of substances causing bronchoconstriction by morphine (9), IgE mediated type I hypersensitivity reaction by penicillin (10) and enzymatic degradation 
Table 1. Drugs causing various patterns of pulmonary parenchymal disorders

\begin{tabular}{|c|c|c|}
\hline Condition & Offending drugs & References \\
\hline $\begin{array}{l}\text { Bronchiolitis obliterans organizing } \\
\text { pneumonia }\end{array}$ & $\begin{array}{l}\text { Minocycline, amphotericin-B, bleomycin, methotrexate, nitrofurantoin, mitomycin, } \\
\text { doxorubicin, acebutolol, amiodarone, ticlopidine, gold, phenytoin, sulfasalazine, } \\
\text { L-tryptophan, interferon }\end{array}$ & 17,18 \\
\hline Hypersensitivity lung disease & $\begin{array}{l}\text { Ampicillin, bupropion, cytarabine, NSAIDs, sirolimus, penicillin, cephalosporins, } \\
\text { phenytoin, carbamazepine, atorvastatin, sulfonamides, ticlopidine, ciprofloxacin, } \\
\text { interferon-alfa, trimethoprim-sulfamethoxazole }\end{array}$ & 22,23 \\
\hline Pulmonary eosinophilia & $\begin{array}{l}\text { Amiodarone, captopril, bleomycin, gold salts, tryptophan, methotrexate, phenytoin, } \\
\text { acetyl-salicylic acid, GM-CSF, minocycline, carbamazepine, penicillamine, } \\
\text { sulfasalazine, propylthiouracil, chloroquine, cocaine, diclofenac, heroin, isoniazid, } \\
\text { isotretinoin, zafirleukast }\end{array}$ & $44-46$ \\
\hline Pulmonary edema & $\begin{array}{l}\text { Cytarabine, amlodipine, heroin, gemcitabine, cyclosporine, verapamil, interleukin, } \\
\text { epinephrine, aspirin, methotrexate, acetazolamide, propoxyphene, ethchlorvynol, } \\
\text { tocolytic agents, propranolol, nitric oxide, chlorthiazide, thiethylperazine, methotrexate, } \\
\text { tretinoin, nitomycin, ketoprofen, heroin, methadone, protamine, clozapine }\end{array}$ & $48-50$ \\
\hline ARDS & $\begin{array}{l}\text { Cytarabine, vinblastine, vincristine, nitrofurantoin, antineoplastic and } \\
\text { immunosuppressant agents, recombinant GM-CSF, infliximab, talc, amiodarone, } \\
\text { immunoglobulin }\end{array}$ & $51-53$ \\
\hline
\end{tabular}

of other drugs in the liver by rifampicin (11). Common causes of drug-induced acute bronchospasm include non-steroidal anti-inflammatory drugs (NSAIDs), aspirin and beta-blockers. Other drugs like angiotensin converting enzyme (ACE) inhibitors, cephalosporins, hydrocortisone, nitrofurantoin, penicillin, and tamoxifen can also cause this manifestation (4). Contrast media used in radiology that contains iodine and the iron dextran compound Imferon may provoke severe anaphylactic responses. Hydrocortisone may cause paradoxically worsening of asthma by unknown mechanism (12). In patients with asthma and chronic obstructive pulmonary disease (COPD) who have a component of reversible airflow limitation, acute bronchospasm with beta-blockers is more likely. However, in one study, patients with COPD and coronary artery disease benefited from $\beta$-blockers; thus, $\beta$-blockers use in patients with COPD is not avoided $(13,14)$. Up to $20 \%$ of asthmatic patients cannot tolerate aspirin and other NSAIDS (15).

\subsection{Bronchiolitis obliterans organizing pneumonia (BOOP)}

Bronchiolitis obliterans is a chronic form of airflow limitation characterized by inflammation and small airways occlusion (16). BOOP is a histopathologic reaction to a nonspecific inflammatory insult and can occur after exposure to a number of drugs $(17,18)$. Symptoms may include nonproductive cough and shortness of breath with bilateral crackles. Imaging shows patchy airspace infiltrates peribronchial or subpleural in location with air trapping. Spirometry may reveal decreased Forced Expiratory Volume (FEV1) and forced expiratory flow at $25 \%$ to $75 \%$ of forced vital capacity (FEV 25-75\%). Diffusing capacity is reduced. Lung biopsy may demonstrate intraluminal buds of granulation tissue with preserved lung architecture. BOOP is treated with drug withdrawal and corticosteroids and has favourable outcome.

\subsection{Hypersensitivity lung disease}

Drug-induced hypersensitivity syndrome (DIHS), also called drug rash with eosinophilia and systemic symptoms (DRESS), is a severe reaction occurring 1-8 weeks after drug introduction. It is an immune mediated reaction involving cytokine and T-lymphocyte activation. Any drug can cause hypersensitivity syndrome. It may present with Loffler syndrome characterized by cough, dyspnea, fever, rash, blood eosinophilia and fleeting pulmonary infiltrates. Hypersensitivity lung disease may also manifest as chronic eosinophilic pneumonia. Patients may present with sub-acute low-grade fever, weight loss and nonproductive cough. Chest radiographs shows patchy peripheral airspace consolidation. Bronchoscopy with bronchoalveolar lavage (BAL) can confirm the diagnosis by revealing eosinophilia (more than $25 \%$ eosinophils). Drug-induced hypersensitivity pneumonitis is a pulmonary syndrome characterized by a complex immunological reaction (19). Patients may present with acute, sub-acute or chronic symptoms of fever, chills, malaise and dyspnea. Spirometry demonstrates a reduced Forced Vital Capacity (FVC) and Diffusion Lung Capacity for carbon monoxide (DLCO). High Resolution Computed Tomography (HRCT) scan Chest may reveal bilateral upper lobepredominant ground-glass opacities, centrilobular nodules and air trapping on expiratory scans. In chronic cases, HRCT may show fibrotic changes, including 
honeycombing and traction bronchiectasis (20). Lung biopsy may demonstrate loosely formed granulomas near the terminal bronchioles and lymphocytic and plasma cell infiltration of the alveolar walls. Pathology may demonstrate a Non-specific interstitial pneumonia (NSIP) or Usual interstitial pneumonia (UIP) pattern (20). Management includes drug withdrawal, but in some cases corticosteroid therapy may be helpful. The common examples of drugs associated with a hypersensitivity pneumonitis include nitrofurantoin and methotrexate (21).

\subsection{Alveolitis}

Most common mechanism of drug-induced alveolitis is allergic reactions. Cyclophosphamide and nitrofurantoin release toxic oxygen species or Acrolein (24). Bleomycin may cause proliferation of fibroblasts and penicillamine may impair collagen formation $(25,26)$. Patients with alveolitis may present with shortness of breath and cough. Chest radiograph shows diffuse patchy infiltration. Drug reaction should be kept in differential diagnosis in all patients presenting with diffuse lung infiltrates. HRCT Chest may reveal early symmetric reticular interstitial markings progressing to fibrotic changes. Pulmonary function tests demonstrate restrictive changes with decreased DLCO and reduced FVC and Total Lung Capacity (TLC). Bleomycin is the most common chemotherapeutic agent, while amiodarone is the most common non-chemotherapeutic agent resulting in alveolitis (27). Phenytoin and carbamazepine are the anticonvulsant drugs that can cause alveolitis. Among cardiac drugs, amiodarone is most frequently associated with alveolitis (28). Amiodarone has a prolonged action and resolution of pulmonary abnormalities occur on withdrawal of drug. Sulfasalazine is the one of the common antiinflammatory drugs associated with alveolitis (29) and the reaction is due to hypersensitivity to the sulphapyridine moiety (30).

\subsection{Drug-induced lupus erythematosus}

Drug-induced lupus erythematosus reactions are most commonly due to hydralazine, procainamide, isoniazid, quinidine, phenytoin and penicillamine (4,31-33). Symptoms begin insidiously after taking the drug for many months. Patients may complain of dyspnea, fever, rash, arthralgias, and joint swelling along with systemic symptoms. Pleuritis presenting with pleural effusion is the most common pulmonary manifestation reported in $50-80 \%$ of patients. In drug-induced lupus erythematosus, antinuclear antibodies or anti-histone antibodies are positive, while anti-double stranded DNA is negative. Chest radiograph may reveal bilateral infiltrates, pneumonitis, atelectasis and/or pleural effusions. Pleural fluid glucose is usually normal.
The treatment involves discontinuation of the drugs. If the drug is used, use in minimal dose along with corticosteroids.

\subsection{Alveolar hemorrhage}

Diffuse alveolar hemorrhage is characterized by the accumulation of red blood cells in the alveolar spaces (34). Patients with alveolar hemorrhage present with hemoptysis, dyspnea, anemia and bilateral infiltrates. Drug induced alveolar hemorrhage may represent a hypersensitivity reaction, an injury to the alveolar capillary basement membrane or a coagulation defect. Drugs that may cause coagulation defects are oral anticoagulants, fibrinolytics and platelet glycoprotein inhibitors (35), drugs resulting in hypersensitivity reaction are propylthiouracil, penicillin, sulfasalazine and hydralazine and drugs having direct toxicity are amiodarone, gefitinib, sirolimus and crack cocaine (36-38). Diagnosis requires bronchoscopy in which serial BAL samples reveal increasingly hemorrhagic fluid along with appearance of new infiltrates on chest radiograph. Lung biopsy is rarely required. Withdrawal of the drug or reversal of a coagulation defect is helpful in alveolar hemorrhage.

\subsection{Interstitial fibrosis}

Inflammation of the lung interstitium is the most common manifestation of DIPPD. Childhood and old age are associated with an increased risk of drug toxicity. Interstitial fibrosis occur when drug reach higher tissue concentrations in the lung by biotransformation process which increases the toxicity by producing reactive metabolites or by consequences of bioactivation which are lung-specific (39). By causing disruption of the oxidant/antioxidant balance, oxygen may precipitate DIPPD (40). Similarly, radiation therapy by synergistic effect with bleomycin can cause lung toxicity. Patients may present with nonproductive cough and dyspnea. HRCT Chest may demonstrate basilar sub-pleural reticular infiltrates; diffuse fibrosis with traction bronchiectasis and honeycombing. Spirometry reveals a restrictive physiology and a reduced DLCO. Lung biopsy demonstrates lymphoid aggregates and plasma cells infiltrating the alveolar septa and the peribronchial spaces. Interstitial fibrosis can also be found (41). In drug-induced NSIP, interstitial inflammation is more homogeneous and cellular than that seen in UIP. This is a progressive disease with poor response to therapy. In acute amiodarone toxicity, mortality may approach 40-50\%, despite drug withdrawal and corticosteroid therapy. Drugs causing pulmonary fibrosis are adalimumab, amiodarone, bleomycin, chlorambucil, cyclophosphamide, gold, interferon, infliximab, methotrexate, nitrofurantoin, paclitaxel, penicillamine, phenytoin, sirolimus and statins. 


\subsection{Eosinophilic lung disease}

Pulmonary manifestations of drug induced eosinophilic lung diseases are simple pulmonary eosinophilia, acute eosinophilic pneumonia, chronic eosinophilic pneumonia, churg-strauss syndrome and eosinophilic pleural effusion. Drug induced eosinophilic lung disease is a diagnosis of exclusion. Thus, history and physical examination are required for the diagnosis. Laboratory investigation is helpful to exclude other diseases. Blood eosinophil count is frequently elevated. Chest radiograph is often helpful to diagnose other lung diseases that may mimic drug induced lung disease. BAL may demonstrate elevated percentage of eosinophils and lymphocytes (42). Eosinophilic lung disease can be suspected by the finding of an increased number of eosinophils in the blood or BAL fluid of a patient who has pulmonary symptoms or infiltrates on chest radiograph. Lung biopsy is nonspecific and rarely required. Symptoms may resolve with discontinuation of the drugs and rarely treatment with corticosteroids is required. Both idiopathic and drug induced acute eosinophilic pneumonia mimic radiologically (43). Minocycline has been reported to cause acute eosinophilic pneumonia (44). Drug induced acute eosinophilic pneumonia recur on re-challenge with offending drug. Churg-Strauss syndrome can be the result of leukotriene inhibitors like zafirlukast and montelukast (45). Whether leukotriene inhibitors cause Churg-Strauss syndrome or their use as a steroid sparing agents in asthmatics allows the development of symptoms of occult Churg-Strauss syndrome is unknown (46). The clinical and radiological presentation of idiopathic and drug induced ChurgStrauss syndrome are similar. Treatment requires longterm corticosteroids and cytotoxic medications.

\subsection{Pulmonary edema and acute respiratory distress syndrome}

Pulmonary edema and Acute Respiratory Distress Syndrome (ARDS) are common manifestations of drug induced lung disease. Clinical features mimic other causes of pulmonary edema. Clinical features may include dyspnea, tachypnea and hypoxemia. Pathogenesis is unknown for most drugs. Idiosyncratic reaction is most common mechanism followed by capillary leak, anaphylaxis and hypervolemia for drug induced pulmonary edema. Increased permeability leads to protein and fluid to enter the lung interstitium and alveolar spaces. Chest radiograph may show alveolar and interstitial infiltrates. Unlike cardiogenic pulmonary edema, cardiomegaly and vascular redistribution are absent. Diagnosis is based on exclusion of other causes. Most reactions are self-limited. Treatment includes oxygen and diuretic therapy. Corticosteroids role is controversial. Positive pressure ventilation may be required in severe cases (47). Symptoms may recur on re-challenge.

\section{Diagnosis}

Establishing a direct relationship between the administration of a drug and the development of lung disease is the first step toward determining drug induced pulmonary parenchymal disease. Drug initiation always precedes clinical symptoms. The time to develop a drug-induced lung parenchymal injury may be quick in the case of bronchospasm, or several years in the case of drug induced pulmonary fibrosis. The diagnostic approach requires review of the patient's presentation and medical history with current and past medication use, including dosages and schedule. There are no definitive symptoms, physical examination, laboratory and radiographic features associated with DIPPD. Acute bronchospasm is characterized by obstructive changes, including a reduced FEV1 and FEV1/ FVC ratio with a significant bronchodilator response. Bronchiolitis obliterans may be suggested by evidence of fixed obstructive lung disease, initially manifested by a reduced FEF1, followed by decreasing FEV25-75 with a low FEV1/ FVC ratio without a bronchodilator response. Pulmonary fibrosis is suggested by a restrictive pattern with a decreased FVC and TLC. Gas exchange is impaired due to ventilation-perfusion mismatching and decreased DLCO due to impaired diffusion across the abnormal interstitium. Arterial blood gas analysis may reveal hypoxemia at rest but should have also arterial oxygen saturation determined during exertion by performing six-minute walk test, because many patients with only mild disease undergo desaturation with exertion despite normal saturation at rest. Complete blood cell count with differential, sputum studies, and serological findings such as rheumatoid factor or anti nuclear antibody and echocardiography are necessary to exclude other causes. Fibreoptic bronchoscopy with BAL may be indicated in case of non-conclusive noninvasive diagnostic measures. BAL findings in drug-induced hypersensitivity pneumonitis include lymphocytosis $>$ 50\% and a decreased CD4 to CD8 ratio. Eosinophilic pneumonia is characterized by an elevated BAL eosinophil count $>25 \%$ (54). BAL findings in alveolar hemorrhage reveal hemosiderin-laden macrophages and an increasingly bloody return from repeated BAL saline aliquots. BAL fluid often has foamy macrophages in patients taking amiodarone. Cytotoxic pneumonitis due to chemotherapeutic agents generally has a neutrophil predominance. Bronchoscopy with negative culture results helps in ruling out infectious causes. But these findings indicate medication exposure and do not necessarily establish drug toxicity. Sensitivity of open lung or surgical biopsy is not high but can be useful in diagnosing hypersensitivity pneumonitis, bronchiolitis obliterans and organizing pneumonia. They can exclude 
the possibility of alternative disease or infection.

\section{Management}

Management of pulmonary drug reactions consists in stopping the offending drug and substituting with less harmful one. Occasionally, corticosteroid therapy may be used. In BOOP, hypersensitivity pneumonitis and eosinophilic pneumonia, corticosteroids can hasten resolution of symptoms, but in pulmonary fibrosis, pulmonary vascular disease and bronchiolitis obliterans, corticosteroids have no role. If allergy is suspected, a challenge test can be carried out after an interval during which the drug is not taken. Hyposensitization, or induction of tolerance, can be done if the drug is essential, all alternatives are unsatisfactory and the reaction is likely to be allergic or idiosyncratic. Clinicians must educate the patients about the potential adverse effects of the drug before starting treatment. Those who develop drug toxicity should be advised to avoid the drug in the future.

\section{Conclusion}

Currently there are several drugs that can be recognized as offending agent for DIPPD. At present, there is no consensus for a definite diagnostic workup approach in patients with a suspicion of DIPPD. Although lung biopsies are not pathognomonic for drug toxicity and correlation with clinical, laboratory, and radiologic data is essential, they can be considered as a powerful tool in the evaluation of suspected by helping to exclude underlying disease or infection and documenting the pattern of parenchymal injury. Therefore, it is important for physicians to be familiar with iatrogenic diseases for which their patients are at risk. The information is helpful in making the diagnosis of drug toxicity as well as guiding the optimal management of the patient in near future.

\section{References}

1. Sternbach G. William Osler: Narcotic-induced pulmonary edema. J Emerg Med. 1983; 1:165-167.

2. Rosenow EC 3rd. Drug-induced pulmonary disease. Dis Mon. 1994; 40:253-310.

3. Ozkan M, Dweik RA, Ahmad M. Drug-induced lung disease. Cleve Clin J Med. 2001; 68:782-785.

4. Raissy HH, Harkins M, Marshik PL. Druginduced pulmonary disease. Pharmacotherapy: A Pathophysiologic Approach. 7th ed., McGraw-Hill, New York, USA, 2008; pp. 521-534.

5. Decalmer PBS, Chatterjee SS, Cruikshank JM, Benson MK, Sterling GM. Beta-blockers and asthma. Br Heart J. 1978; 40:184-189.

6. Fratto C. Provocation of bronchospasm by eye drops. Ann Intern Med. 1978; 88:362.

7. Sanak M, Simon HU, Szczeklik A. Leukotriene C4 synthase promoter polymorphism and risk of aspirin- induced asthma. Lancet. 1997; 350:1599.

8. Connolly CK. Adverse reaction to ipratropium bromide. Br Med J. 1982; 285:934.

9. Gilman AG, Goodman LS, Rall TW, Murad F. The Pharmacological Basis of Therapeutics. 7th ed., Macmillan, New York, USA, 1985; p. 507.

10. Coutts II, Dally MB, Newman Taylor AJ. Asthma in workers manufacturing cyclosporins. Br Med J. 1981; 283:950.

11. Powell-Jackson PR, Gray BJ, Heaton RW, Costello JF, Williams R, English J. Adverse effect of rifampicin administration on steroid-dependent asthma. Am Rev Respir Dis. 1983; 128:307.

12. Dajani BM, Sliman NA, Shubair KS, Hamzeh YS. Bronchospasm caused by intravenous hydrocortisone sodium succinate (Solu-Cortef) in aspirin sensitive subjects. J Allergy Clin Immunol. 1984; 68:201.

13. Salpeter S, Ormiston T, Salpeter E. Cardioselective betablocker use in patients with reversible airway disease. Cochrane Database Syst Rev. 2001; 2:CD002992.

14. Au DH, Bryson CL, Fan VS, Udris EM, Curtis JR, McDonell MB, Fihn SD. Beta-blockers as single-agent therapy for hypertension and the risk of mortality among patients with chronic obstructive pulmonary disease. Am J Med. 2004; 117:925-931.

15. Babu K, Salvi S. Aspirin and asthma. Chest. 2000; 118:1470-1476.

16. Visscher DW, Myers JL. Bronchiolitis: The pathologist's perspective. Proc Am Thorac Soc. 2006; 3:41-47.

17. Cordier JF. Organizing pneumonia. Thorax. 2000; 55:318-328.

18. Erasmus JJ, McAdams HP, Rossi SE. Drug induced lung injury. Sem Roentgen. 2002; 37:72-81.

19. Bourke SJ, Dalphin JC, Boyd G, McSharry C, Baldwin CI, Calvert JE. Hypersensitivity pneumonitis: Current concepts. Eur Respir J Suppl. 2001; 32:81S-92S.

20. Silva CI, Churg A, Muller NL. Hypersensitivity pneumonitis: Spectrum of high-resolution CT and pathologic findings. AJR. 2007; 188:334-344.

21. Louie S, Lillington GA. Low dose methotrexate pneumonitis in rheumatoid arthritis. Thorax. 1986; 41:703-704.

22. Lantuejoul S, Brambilla E, Brambilla C, Devouassoux G. Statin-induced fibrotic nonspecific interstitial pneumonia. Eur Respir J. 2002; 19:577-580.

23. Schwaiblmair M, Behr W, Haeckel T, Märkl B, Foerg W, Berghaus T. Drug-induced infiltrative lung disease: Eur Respir J. 2001; 18:93s-100s

24. Martin WJ. Nitrofurantoin: Evidence for the oxidant injury of lung parenchymal cells. Am Rev Respir Dis. 1983; 127:482.

25. Kelley J, Newman RA, Evans JN. Bleomycin-induced pulmonary fibrosis in the rat. Prevention with an inhibitor of collagen. J Lab Clin Med. 1980; 96:954.

26. Nimni ME, Bavetta LA. Collagen defect induced by penicillamine. Science. 1965; 150:905.

27. Camus P, Bonniaud P, Fanton A, Camus C, Baudaun N, Foucher P. Drug-induced and iatrogenic infiltrative lung disease. Clin Chest Med. 2004; 25:479-519.

28. Roca J, Heras M, Rodriquez-Roisin R, Magrina J, Xaubet A, Sanz G. Pulmonary complications after longterm amiodarone treatment. Thorax. 1992; 47:372.

29. Averbuch M, Halpern Z, Hallak A, Topilsky M, Levo Y. Sulfasalazine pneumonitis. Am J Gastroenterol. 1985; 80:343. 
30. Thomas P, Seaton A, Edwards J. Respiratory disease due to sulphasalazine. Clin Allergy. 1974; 4:41.

31. Bhadra K, Suratt B. Drug-induced lung disease: A stateof-the-art review. J Resp Dis. 2009; 30:1-17.

32. Foucher P, Biour M, Blayac JP, Godard P, Sgro C, Kuhn $\mathrm{M}$, Vergnon JM, Vervloet D, Pfitzenmeyer P, Ollagnier M, Mayaud C, Camus P. Drugs that may injure the respiratory system. Eur Respir J. 1997; 10:265-279.

33. Birnbaum B, Sidhu GS, Smith RL, Pillinger MH, Tagoe CE. Fulminating hydralazine-induced lupus pneumonitis. Arthritis Rheum. 2006; 55:501-506.

34. Fontenot AP, Schwarz MI. Diffuse alveolar hemorrhage. Interstitial Lung Disease. 2003; 3:632-656.

35. Kalra S, Bell MR, Rihal CS. Alveolar hemorrhage as a complication of treatment with abciximab. Chest. 2001; 120:126-131.

36. Vlahakis NE, Rickman OB, Morgenthaler T. Sirolimusassociated diffuse alveolar hemorrhage. Mayo Clin Proc. 2004; 79:541-545.

37. Janjua TM, Bohan AE, Wesselius LJ. Increased lower respiratory tract iron concentrations in alkaloidal (“crack") cocaine users. Chest. 2001; 119:422-427.

38. Leki R, Saitoh E, Shibuya M. Acute lung injury as a possible adverse drug reaction related to gefitinib. Eur Respir J. 2003; 22:179-181.

39. Schwaiblmair M, Berghaus T, Haeckel T, Wagner T, von Scheidt W. Amiodarone induced pulmonary toxicity: An underrecognized and severe adverse effect? Clin Res Cardiol. 2010; 99:693-700.

40. Bast A, Weseler A, Haenen G, den Hartog G. Oxidative stress and antioxidants in interstitial lung disease. Curr Opin Pulm Med. 2010; 16:516-520.

41. Huggett M, Armstrong R. Adalimumab-associated pulmonary fibrosis. Rheumatology. 2006; 45:1312-1313.

42. Akoun GM, Cadranel JL, Milleron BJ, D'Ortho MP, Mayaud CM. Bronchoalveolar lavage cell data in 19 patients with drug associated pneumonitis (except amiodarone). Chest. 1991; 99:98-104.

43. Yokoyama A, Mizushima Y, Suzuki H, Arai N, Kitagawa M, Yano S. Acute eosinophilic pneumonia induced by minocycline: Prominent kerley B lines as a feature of positive re-challenge test. Jpn J Med. 1990; 29:195-198.

44. Dussopt C, Mornex JF, Cordier JF, Brune J. Acute eosinophilic lung after a course of minocycline. Rev Mal Respir. 1994; 11:67-70.

45. Knoell DL, Lucas J, Allen JN. Churg-Strauss syndrome associated with zafirlukast. Chest. 1998; 114:332-334.

46. Weller PF, Plaut M, Taggart V, Trontell A. The relationship of asthma therapy and Churg-Strauss syndrome: NIH workshop summary report. J Allergy Clin Immunol. 2001; 108:175-183.

47. Reed CR, Glauser FL. Drug-induced non-cardiogenic pulmonary edema. Chest. 1991; 100:1120-1124.

48. Gallerani M, Manzoli N, Fellin R, Simonato M, Orzincolo C. Anaphylactic shock and acute pulmonary edema after a single oral dose of acetazolamide. Am J Emerg Med. 2002; 20:371-372.

49. Conces DJ, Kreipke DL, Tarver RD. Pulmonary edema induced by intravenous ethchlorvynol. Am J Emerg Med. 1986; 4:549-551.

50. Tweeddale MG. Salicylates and pulmonary edema. Ann Intern Med. 1974;81:710-711.

51. Riegert-Johnson DL, Godfrey JA, Myers JL, Hubmayr RD, Sandborn WJ. Delayed hypersensitivity reaction and acute respiratory distress syndrome following infliximab infusion. Inflamm Bowel Dis. 2002; 8:186-191.

52. Verhoef G, Boogaerts M. Treatment with granulocytemacrophage colony stimulating factor and the adult respiratory distress syndrome. Am J Hematol. 1991; 36:285-287.

53. Van Mieghem W, Coolen L, Malysse I, Lacquet LM, Deneffe GJ. Amiodarone and the development of ARDS after lung surgery. Chest. 1994; 105:1642-1645.

54. Janz DR, O'Neal HR Jr, Ely EW. Acute eosinophilic pneumonia: A case report and review of the literature. Crit Care Med. 2009; 37:1470-1474.

(Received July 7, 2014; Revised December 21, 2014; Accepted December 21, 2014) 